\title{
ADAPTABILIDAD Y ESTABILIDAD DE 20 VARIEDADES DE MAÍZ, PANAMÁ ${ }^{1}$
}

\author{
Román Gordón-Mendoza $a^{2}$, Jorge Franco-Barrera ${ }^{2}$, Ismael Camargo-Buitrago ${ }^{3}$
}

\section{RESUMEN}

Adaptabilidad y estabilidad de 20 variedades de maíz, Panamá. El objetivo de este trabajo fue determinar la adaptabilidad y estabilidad de variedades de maíz en Panamá. Durante el periodo de setiembre del 2007 a enero del 2008, se evaluaron ensayos uniformes de rendimiento en seis localidades con ambientes contrastantes en Panamá. El material genético de este ensayo consistió de nueve sintéticos QPM y once de grano normal, provenientes del CIMMYT. Se utilizó un diseño experimental de bloques completos al azar con tres repeticiones, las parcelas experimentales consistieron de dos surcos de 5,0 $\mathrm{m}$ de largo, separadas a $0,75 \mathrm{~m}$. El análisis de varianza combinado mostró diferencias estadísticas significativas entre ambos grupos (normal vs QPM) y dentro de cada grupo para el rendimiento y las otras variables de importancia económica y genética. Por otro lado, se encontró que la interacción genotipo por ambiente fue significativa para el rendimiento de grano indicando una respuesta diferencial de los genotipos. El análisis combinado de las medias de rendimiento mostró que entre los sintéticos normales se destacaron el SA-N6-07, SA-N8-07 y SA-N707 con promedios superiores a las 4,0 t/ha, mientras que en los QPM sobresalieron el SA-Q14-07 y SA-Q12-07 con rendimientos de 3,98 y 3,63 t/ha. Todos estos sintéticos superaron tanto al testigo de grano normal (Guararé-8128) como al testigo QPM (SA-Q5-06) que tuvieron rendimientos de 2,93 y 3,36 t/ha, respectivamente. El modelo Biplot GGE-SREG, identificó los sintéticos SA-N6-07, SA-Q1407, SA-N4-07 y SA-N10-07 como los de mejor estabilidad; adicional los dos primeros fueron los mejores en rendimiento para cada tipo de grano.

\begin{abstract}
Adaptability and stability of 20 corn varieties in Panama. The objective of this work was to determine the adaptability and stability of 20 corn varieties in Panama. From September 2007 to January 2008, uniform yield tests were evaluated in six locations with contrasting environments in Panama. The genetic material consisted of nine QPM synthetics and eleven with normal grains, all from CIMMYT. The experimental design employed was complete randomized blocks with three replications. The experimental plots were two 5-m long rows with $0,75 \mathrm{~m}$ between rows. The combined analysis of variance showed statistically significant differences between the two groups (synthetic vs. normal) for yield and other economically and genetically important variables within groups. On the other hand, the interaction genotype $\mathrm{X}$ environment was significant for grain yield, indicating differential responses of the genotypes. The combined analysis of the mean yields showed that among the normal synthetics, SA-N6-07, SA-N8-07 and SA-N707 exhibited outstanding yield with means higher than 4,0 t/ha, whereas within the QPM, SA-Q14-07 and SA-Q12-07 yielded 3,98 and 3,63 t/ha. The yield of a all these synthetics surpassed the normal grain control (Guararé-8128) and the QPM control (SA-Q5-06) which yielded 2.93 and 3.36 t/ha, respectively. The Biplot GGE-SREG model identified the synthetics SA-N6-07, SA-Q14-07 and SA-N10-07 with the highest stability; additionally, the first two were the best yielding varieties within each group.
\end{abstract}

Key words: QPM, normal grain, yellow grain, Biplot GGE-SREG.

Palabras claves: QPM, grano normal, grano amarillo, Biplot GGE-SREG.

\footnotetext{
Recibido: 28 de junio, 2009. Aceptado: 17 de mayo, 2010. Investigación realizada con fondos de la Secretaría Nacional de Ciencia, Tecnología e Innovación de Panamá bajo el contrato CCP06-033.

2 Instituto de Investigación Agropecuaria de Panamá, Centro de Investigación Agropecuaria de Azuero, "Ing. Germán De León”, Los Santos, Panamá. gordon.roman@gmail.com; joenfra13@gmail.com

3 Instituto de Investigación Agropecuaria de Panamá, Centro de Investigación Agropecuaria en Recursos Genéticos "Ing. Alfonso Alvarado", Río Hato, Panamá. icamargo@cwpanama.net
} 


\section{INTRODUCCIÓN}

En el maíz hay principalmente cuatro clases de proteínas: globulina, albúmina, prolamina (zeína) y glutelina. La zeína es una proteína de pobre calidad y constituye más de la mitad del contenido de proteína del maíz normal. El gen Opaco-2 disminuye el contenido de zeína en el grano hasta en un $50 \%$ y aumenta los niveles de lisina y triptófano. El maíz con alta calidad de proteínas también llamado QPM, por sus siglas en inglés (Quality Protein Maize) es portador del gen Opaco-2, es decir son ricos en lisina y triptófano, contando con el doble de unidades que los maíces normales (FAO 1993, Krivanek et al. 2007, Mendoza et al. 2006).

A partir del descubrimiento del gen Opaco-2 por científicos de la Universidad de Purdue en 1963 y los trabajos realizados posteriormente para generar nuevas variedades con este gen; hubo un aparente desinterés en la adopción de cultivares de maíces QPM debido al poco atractivo del grano, a la susceptibilidad a factores bióticos y abióticos tanto en campo, como en el almacenamiento y al bajo rendimiento que presentaron las primeras variedades que portaban este gen. A partir de inicios de los años 1970, los fitomejoradores del Centro Internacional de Mejoramiento de Maíz y Trigo, apoyados por el laboratorio de bioquímica de esa institución, continuaron mejorando las características agronómicas y nutricionales de estos maíces (CIMMYT 2009, Ortega et al. 2001). En los primeros años de la década de los 90 presentaron al mundo científico variedades de maíz con alta calidad nutritiva, altos rendimientos, humedad normal en el grano y aspecto de grano duro normal (no harinoso). Para 1996, el maíz Opaco-2 había sido modificado a lo que se podría llamar un maíz tipo "normal" en todas sus características, excepto por el valor nutricional de su proteína (CIMMYT 1999). Hoy en día estos nuevos materiales son considerados como una de las estrategias para mitigar la desnutrición en zonas de pobreza y alta desnutrición.

En las áreas urbanas de nuestro país, la pobreza total y la extrema alcanzan su menor nivel, el 20,6\% y $4,6 \%$ de su población, respectivamente. En tanto que, en las áreas rurales no indígenas, poco más de la mitad de los residentes es pobre $(54,2 \%)$, y una de cada cinco personas $(22,3 \%)$ se encuentra en situación de pobreza extrema. En las áreas rurales indígenas esta situación se empeora, en donde casi la totalidad de sus habitantes se encuentra en condición de pobreza $(98,5 \%)$, mientras que el $89,7 \%$ de sus pobladores se encuentra en pobreza extrema (MEF 2005).

El análisis de varianza y regresión conjunta, es una metodología empleada ampliamente para explicar la interacción G x A (Finlay y Wilkinson 1963, Eberhart y Russell 1966, Perkins y Jinks 1968). Técnicas multivariadas también han sido usadas para estudiar los efectos de la interacción $\mathrm{G}$ x A; por ejemplo el análisis de componentes principales (PCA), análisis de coordenadas principales, y análisis de clúster (Crossa 1990, Westcott 1986). El desarrollo del modelo AMMI (Efectos principales aditivos e interacción multiplicativa), que integra análisis de varianza y de componentes principales (Zobel et al. 1988), ha mostrado su eficiencia para explicar una proporción de la suma de cuadrados de la interacción, superior a la obtenida con el análisis de varianza y regresión conjunta (Gauch y Zobel 1988, Zobel et al. 1988, Crossa 1988, Crossa 1990, Crossa et al. 1990 y Crossa et al. 1991).

Independientemente de la metodología empleada para estimar la interacción G x A, hay que tener claro a cual concepto de estabilidad se refiere. Becker (1981), Lin et al. (1986), Becker y León (1988), definen conceptos de estabilidad fenotípica que se complementan del punto de vista estadístico, biológico y agronómico. El objetivo de este trabajo fue determinar la adaptabilidad y estabilidad de variedades de maíz en Panamá.

\section{MATERIALES Y MÉTODOS}

\section{Ubicación}

Se realizó un experimento en seis localidades de la República de Panamá, en parcelas facilitadas por pequeños productores de maíz de las provincias de Panamá, Darién, Los Santos, Veraguas y la Comarca Ngöbe Bugle, durante la segunda época de siembra (septiembre 2007 a enero del 2008) (Cuadro 1). Todas estas localidades están ubicadas a menos de 300 msnm.

\section{Caracterización edáfica}

Antes de la siembra de los ensayos se tomaron varias muestras de suelo en cada localidad para su análisis físico-químico. Las mismas se tomaron en cada uno de los bloques a una profundidad de 0 a $20 \mathrm{~cm}$. Estas 
Cuadro 1. Ubicación, fecha de siembra y cosecha de las seis localidades del ensayo de evaluación de sintéticos de maíz. Instituto de Investigación Agropecuaria de Panamá (IDIAP), Panamá, 2007-2008.

\begin{tabular}{|c|c|c|c|c|c|c|c|}
\hline & Localidad & Provincia & Distrito & Latitud & Longitud & Fecha de siembra & Fecha de cosecha \\
\hline 1. & Santa Fe & Darién & Tortí & $8^{\circ} 39^{\prime}$ & $79^{\circ} 16^{\prime}$ & $25 \mathrm{Sep}$ & $13 \mathrm{Feb}$ \\
\hline 2. & Ollas Arriba & Panamá & Capira & $8^{\circ} 48^{\prime}$ & $79^{\circ} 53^{\prime}$ & 19-Sep & 28-Ene \\
\hline 3. & Chupá & Los Santos & Macaracas & $7^{\circ} 40^{\prime}$ & $80^{\circ} 29^{\prime}$ & 18-Sep & 23-Ene \\
\hline 4. & El Ejido & Los Santos & Los Santos & $7^{\circ} 54^{\prime}$ & $80^{\circ} 22^{\prime}$ & 31-Ago & 29-Ene \\
\hline 5. & La Zumbona & Veraguas & Soná & $8^{\circ} 08^{\prime}$ & $80^{\circ} 42^{\prime}$ & 28-Sep & $15-\mathrm{Feb}$ \\
\hline 6. & Llano Jengibre & Ngöbe Bugle & Mironó & $8^{\circ} 20^{\prime}$ & $81^{\circ} 60^{\prime}$ & 21-Sep & 16-Ene \\
\hline
\end{tabular}

fueron homogenizadas y enviadas al Laboratorio de Suelos del Instituto de Investigación Agropecuaria de Panamá (IDIAP), en donde se realizó el análisis según Díaz-Romeu y Hunter (1978). El análisis de suelo de los sitios mostró una alta variabilidad; en donde se encontraron suelos desde muy ácidos ( $\mathrm{pH} 4,8)$ a neutros ( $\mathrm{pH} 7,1)$, con texturas que van de franco-arenosa hasta arcillosa. En cuanto a la fertilidad todos son bajos en fósforo con excepción de Santa Fe, de contenido medio en potasio, bajos en aluminio y de medio a altos en calcio y magnesio. El porcentaje de materia orgánica osciló entre 1,5 a 7,6\% (Cuadro 2).

\section{Material genético}

El grupo de maíces sintéticos evaluados fueron obtenidos del Centro Internacional de Mejoramiento de Maíz y Trigo (CIMMYT) con sede en El Batán, Texcoco, México. El ensayo incluyó 10 genotipos experimentales de grano color amarillo normal y ocho de color de grano amarillo de alta calidad proteica. Se incluyeron dos testigos, uno normal (Guararé 8128) y otro de alta calidad proteica (SA-Q5-06) (Cuadro 3).

Según Cubero 2003, las variedades sintéticas son aquellas formadas por el cruzamiento de un cierto número de parentales (en principio líneas), elegidos para que tengan buena aptitud combinatoria general. El número de líneas envueltas en la formación de los sintéticos varía en un número menor a 20 . Se diferencia de una variedad de polinización libre tradicional en que esta última está compuesta por un número mucho mayor de parentales.

\section{Manejo agronómico}

El sistema de preparación del suelo en todos los experimentos fue el de cero labranza con excepción del ubicado en El Ejido. La densidad inicial de siembra en las localidades de Santa Fe, Ollas Arriba, La Zumbona y Llano Jengibre fue de 5,33 plantas $/ \mathrm{m}^{2}$, ésto se logró sembrando surcos separados a $0,75 \mathrm{~m}$ y se dejaron dos plantas cada 0,50 m. En las localidades de El Ejido y Chupá

Cuadro 2. Principales características físico químicas de los suelos de los ensayos de sintéticos de maíz amarillo normal y QPM en las seis localidades del ensayo de evaluación. Instituto de Investigación Agropecuaria de Panamá (IDIAP), Panamá, 2007 2008.

\begin{tabular}{|c|c|c|c|c|c|c|c|c|c|c|c|c|c|c|c|}
\hline \multirow[t]{2}{*}{ Localidad } & Are & Lim & Arc & \multirow[t]{2}{*}{ pH } & \multirow{2}{*}{$\begin{array}{c}\mathbf{P} \\
(\mu \mathrm{g} / \mathrm{ml})\end{array}$} & $\mathbf{K}$ & $\mathbf{C a}$ & Mg & Al & \multirow{2}{*}{$\begin{array}{c}\text { M.O. } \\
\text { (\%) }\end{array}$} & Mn & $\mathbf{F e}$ & Zn & $\mathbf{C u}$ & \multirow[t]{2}{*}{ Textura* } \\
\hline & & (\%) & & & & \multicolumn{4}{|c|}{ (meq/100ml) } & & \multicolumn{4}{|c|}{$(\mu \mathrm{g} / \mathrm{ml})$} & \\
\hline El Ejido & 44 & 16 & 40 & 4,9 & $\operatorname{tr}$ & 0,09 & 10,0 & 8,0 & 0,2 & 2,8 & 11 & $\operatorname{tr}$ & $\operatorname{tr}$ & 6 & $\mathrm{ARC}$ \\
\hline Chupá & 46 & 24 & 30 & 4,8 & 1 & 0,19 & 9,9 & 6,5 & 0,1 & 4,6 & 16 & $\operatorname{tr}$ & 1 & 6 & FARA \\
\hline La Zumbona & 66 & 18 & 16 & 5,7 & $\operatorname{tr}$ & 0,49 & 5,9 & 2,1 & 0,1 & 7,6 & 20 & 4 & $\operatorname{tr}$ & $\operatorname{tr}$ & FA \\
\hline Llano Jengibre & 70 & 20 & 8 & 4,9 & 2 & 0,47 & 5,0 & 4,5 & 0,6 & 2,6 & 16 & 11 & 1 & 3 & FARA \\
\hline Ollas Arriba & 54 & 12 & 34 & 5,4 & $\operatorname{tr}$ & 0,12 & 2,4 & 4,5 & 0,1 & 5,2 & 32 & 32 & 1 & 3 & FARA \\
\hline Santa Fe & 38 & 22 & 40 & 7,1 & 17 & 0,35 & 24,0 & 2,6 & 0,1 & 1,5 & 20 & 8 & 3 & 1 & ARC \\
\hline
\end{tabular}

$* \mathrm{FA}=$ franco arenoso, FARA $=$ franco arcillo-arenoso, $\mathrm{ARC}=$ arcilloso, $\operatorname{tr}=$ trazas 
Cuadro 3. Nombre, tipo del grano y genealogía de los sintéticos de maíz normales y QPM evaluados en el Instituto de Investigación Agropecuaria de Panamá (IDIAP), Panamá. 2007-2008.

\begin{tabular}{llll}
\hline & Nombre & \multicolumn{1}{c}{$\begin{array}{c}\text { Tipo del } \\
\text { grano }\end{array}$} & \multicolumn{1}{c}{ Pedigrí } \\
\hline 1. & SA-N1-07 & Normal & S03TLY-3 AB \\
2. & SA-N2-07 & Normal & S03TLY-4 AB \\
3. & SA-N3-07 & Normal & S03TLY-5 AB \\
4. & SA-N4-07 & Normal & S03TLY- LN \\
5. & SA-N5-07 & Normal & S04TLY-1 \\
6. & SA-N6-07 & Normal & S04TLY-2 \\
7. & SA-N7-07 & Normal & S03TLY-LN/Pp \\
8. & SA-N8-07 & Normal & S00TLY-1 AB \\
9. & SA-N9-07 & Normal & S00TLY-Bm/Pp \\
10. & SA-N10-07 & Normal & S03TLY-2 AB \\
11. & SA-Q11-07 & QPM & S05TLYQ-HG-AB-1 \\
12. & SA-Q12-07 & QPM & S05TLYQ-HG-AB-2 \\
13. & SA-Q13-07 & QPM & S05TLYQ-HG-A \\
14. & SA-Q14-07 & QPM & S05TLYQ-HG-B \\
15. & SA-Q1-06 & QPM & S 03 TLYQ- AB 02 \\
16. & SA-Q3-06 & QPM & S 03 TYLQ-AB 01 \\
17. & SA-Q17-07 & QPM & S03TLYQ-AB03 \\
18. & SA-Q2-06 & QPM & S 99 TLYQ-AB(RE) \\
19. & Guararé 8128 & Normal & \\
20. & SA-Q5-06 & QPM & S 03 TLYQ-AB 05 \\
\hline & & &
\end{tabular}

la densidad inicial de siembra fue de 6,66 plantas $/ \mathrm{m}^{2}, \mathrm{y}$ se utilizó un arreglo de $0,75 \mathrm{~m}$ entre hileras y $0,20 \mathrm{~m}$ entre plantas. La fertilización consistió en la aplicación de $136 \mathrm{~kg} / \mathrm{ha}$ de la fórmula completa 13-26-6-7 al momento de la siembra, en forma de postura enterrada a cuatro centímetros de la semilla. Posteriormente, se realizaron dos aplicaciones suplementarias de urea, la primera a los 20 días después de siembra (dds) a razón de $45 \mathrm{~kg} / \mathrm{ha} \mathrm{y}$ la segunda a los 37 dds a razón de $91 \mathrm{~kg} / \mathrm{ha}$.

El control de malezas consistió en la aplicación en pre-emergencia de la mezcla de atrazina más pendimentalina a razón de 1,50 y 1,65 kg i.a./ha, respectivamente. En algunas localidades, por la presencia de malezas de mayor tamaño, se añadió a la mezcla el herbicida glifosato a razón de 1,84 kg i.a./ha.

\section{Variables medidas}

Los datos tomados incluyeron caracteres cuantitativos, que son influenciados por el ambiente como: altura de planta y mazorca, rendimiento de grano, porcentaje de mazorcas podridas; $y$, caracteres cualitativos pocos influenciados como: días a floración femenina, número de plantas y mazorcas al momento de la cosecha, porcentaje de plantas acamadas, humedad del grano, aspecto de planta y mazorca y la evaluación de las principales enfermedades al follaje (Curvularia $s p$ y Helminthosporium $\mathrm{sp}$ ) con una escala de una a cinco en donde uno es ausencia de la enfermedad y cinco la planta completamente enferma.

\section{Diseño experimental}

El tamaño de las parcelas experimentales consistió de dos surcos de 5,0 m de largo. Para la ejecución en campo se utilizó un diseño de Bloques Completos al Azar (DBCA), con tres repeticiones, de acuerdo al siguiente modelo matemático:

$$
\mathrm{X}_{\mathrm{IJL}}=\mu+\mathrm{G}_{\mathrm{I}}+\mathrm{B}_{\mathrm{J} / \mathrm{L}}+\mathrm{A}_{\mathrm{L}}+(\mathrm{G} \mathrm{A})_{\mathrm{I} L}+\mathrm{e}_{\mathrm{IJ}}
$$

En donde:

$\mathrm{X}=$ Valor del carácter estudiado

$\mathrm{A}_{\mathrm{L}}=$ Efecto de ambiente

$\mu=$ Media general biente

$(\mathrm{G} A)_{\mathrm{IL}}=$ Efecto de la interacción genotipo am-

$\mathrm{G}_{\mathrm{I}}=$ Efecto de genotipo

$\mathrm{e}_{\mathrm{IJ}}=$ Error experimental

$\mathrm{B}_{\mathrm{J} / \mathrm{L}}=$ Efecto de bloques dentro de repetición

\section{Análisis estadístico}

Se realizó un análisis de varianza combinado, con base en un modelo mixto (ambiente aleatorio y genotipo fijo). Se separó el efecto de la suma de cuadrados de los Genotipos Normales y QPM así como la diferencia entre ambos grupos. Para la separación de medias de todas las variables se utilizó la diferencia mínima significativa (DMS). Para el análisis estadístico de las variables porcentaje de plantas acamadas y porcentaje de mazorcas podridas se utilizó la transformación por el método de la raíz cuadrada más un medio $\sqrt{x+0,5}$.

Para el análisis de estabilidad se utilizó el modelo AMMI Bi-plot GGE-SREG, que integra el análisis de varianza y el análisis de componentes principales (Zobel et al. 1988, Yan et al. 2000). El modelo matemático es:

$$
\mathrm{Y}_{\mathrm{ge}}=\mu+\alpha_{\mathrm{g}}+\beta_{\mathrm{e}}+\Sigma^{\mathrm{N}} \lambda_{\mathrm{n}} \mathrm{Y}_{\mathrm{gn}} \delta_{\mathrm{en}}+Q_{\mathrm{ge}}
$$


En donde:

$\mathrm{Y}_{\mathrm{g} \text { e }}=$ Rendimiento promedio de un genotipo $\mathrm{g}$ en un ambiente e

$\alpha_{\mathrm{g}}=$ Efecto de las desviaciones de las medias de los genotipos

$\mu=$ Media general

$\beta_{\mathrm{e}}=$ Efecto de las desviaciones de las medias del ambiente

$\lambda_{n}=$ Es el valor singular para el PCA

$\delta_{\text {en }}=$ Valores de los vectores para cada ambiente (PCA)

$\mathrm{N}=$ Número de PCA retenidos en el modelo

$\varrho_{\mathrm{ge}}=$ Residual

$\mathrm{Y}_{\mathrm{g} \mathrm{n}}^{\mathrm{ge}}=$ Valores de vectores de los genotipos (PCA)

\section{RESULTADOS Y DISCUSIÓN}

\section{Análisis de varianza}

En el análisis de varianza combinado del rendimiento hubo una diferencia altamente significativa $(\mathrm{P}<$ $0,01)$ entre ambientes para todas las variables estudiadas, y diferencias altamente significativas para el genotipo para todas las variables excepto la altura de plantas y la posición de la mazorca. La interacción genotipoambiente resultó altamente significativa para todas las variables estudiadas con excepción de las variables altura de las plantas, número de las plantas y número de mazorcas $/ \mathrm{m}^{2}$, número de mazorcas por planta, aspecto de las mazorcas y posición de las mazorcas.

En cuanto a los tipos de granos hubo diferencias altamente significativas entre los dos grupos para las variables peso y pudrición de las mazorcas, rendimiento de grano y aspecto de mazorcas. Dentro de cada grupo (normales y QPM) se encontraron diferencias significativas para la variable rendimiento y otras características agronómicas.

\section{Efecto de ambientes}

En este experimento el ambiente capturó el 75,9\% de la Suma de Cuadrados Total del análisis de varianza, para la variable rendimiento de grano. Esto indica, que los ambientes fueron diferentes y contrastantes, con diferencias en la magnitud de las respuestas entre las medias ambientales. El rendimiento de grano y algunas de las variables medidas por localidad se presentan en el Cuadro 4. El rendimiento promedio a través de las seis localidades evaluadas fue de 3,61 t/ha, pero el mismo se vio afectado por la calidad del suelo de las distintas localidades. Los rendimientos más altos se observaron en El Ejido y Chupá con rendimientos de 5,50 y 4,61 t/ha, respectivamente. Esta respuesta está asociada al mayor número de plantas cosechadas en estas dos localidades. A este grupo le siguieron La Zumbona y Llano Jengibre, con rendimientos de 3,89 y 3,58 t/ha. Un tercer grupo estuvo conformado por Santa Fe y Ollas Arriba con rendimientos por debajo de las 3,0 t/ha. El Ejido presentó los mayores porcentajes de plantas acamadas debido al mayor número de plantas cosechadas y la cosecha tardía del mismo (151 días).

El porcentaje de mazorcas podridas a través de las seis localidades fue de 12,6\%, en La Zumbona, Llano Jengibre, Ollas Arriba y Santa Fe se encontraron los mayores porcentajes (superiores al 10\%), mientras que en las localidades de la región de Azuero (El Ejido y Chupá) el valor estuvo por debajo del 5\%, lo que se explica debido a que en éstas la precipitación fue menor al momento del llenado de grano. En relación al peso de las mazorcas, en las localidades de El Ejido, Chupá, Llano Jengibre y La Zumbona se encontraron los mayores valores (más de $80 \mathrm{~g}$ ), mientras que en las dos restantes los valoren no superaron los $70 \mathrm{~g}$. El número de plantas a la cosecha varió a través de las localidades encontrándose las poblaciones más altas en Chupá y El Ejido, en las otras localidades la población de plantas al momento de la cosecha fue menor de 5,2 plantas/m. La baja población de plantas en las localidades de Llano Jengibre, Santa Fe y Ollas Arriba se debió al exceso de humedad en los días siguientes a la siembra, lo que se reflejó en una baja germinación de semillas y por consiguiente en una baja población inicial de plantas.

\section{Efecto de genotipos}

El análisis de varianza para genotipo, mostró diferencias altamente significativas entre los distintos sintéticos evaluados para la variable rendimiento de grano; se logró capturar el 7,2\% de la Suma de Cuadrados Total del análisis de varianza de esta variable. El Cuadro 5 muestra la media de rendimiento de los 20 sintéticos, así como el resto de las características agronómicas más importantes. 


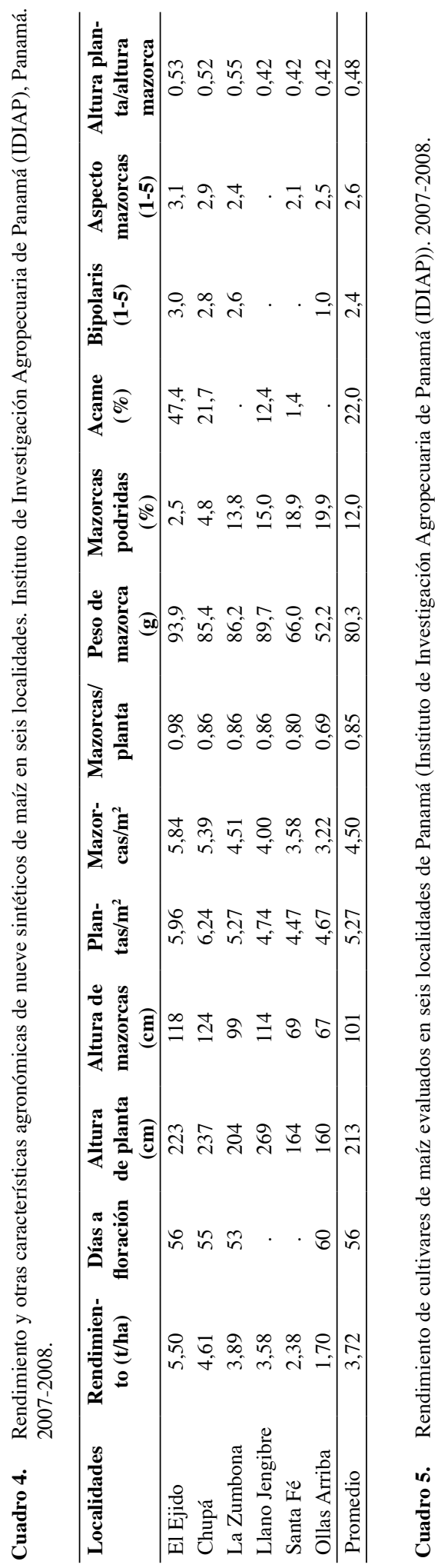

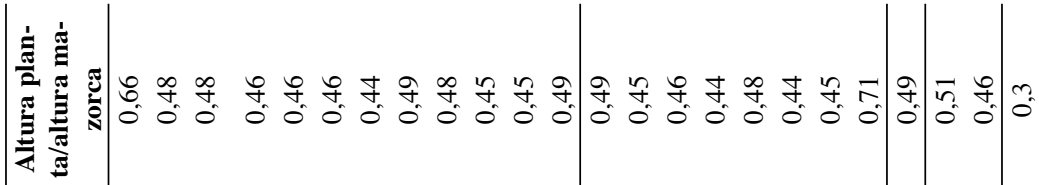

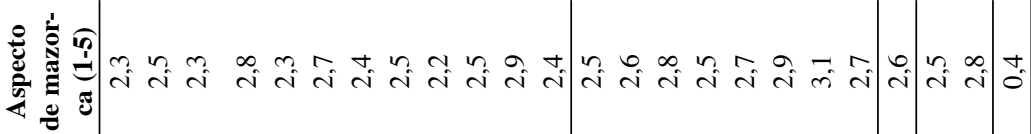

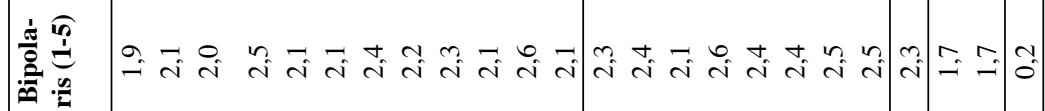
产

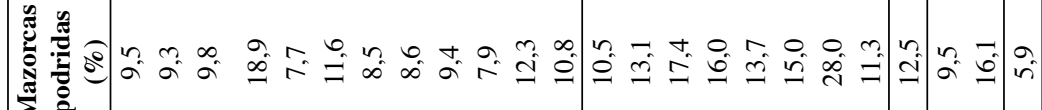

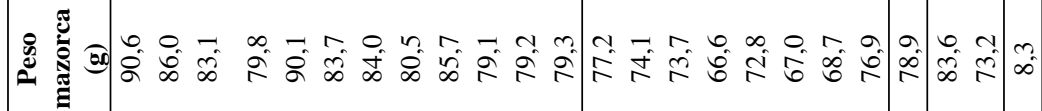

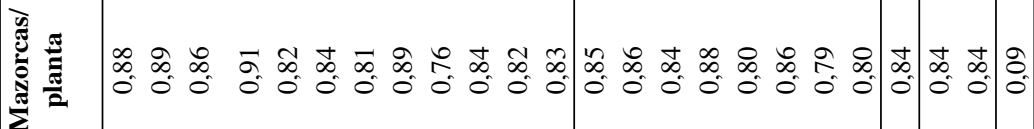

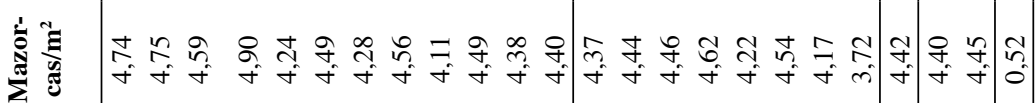

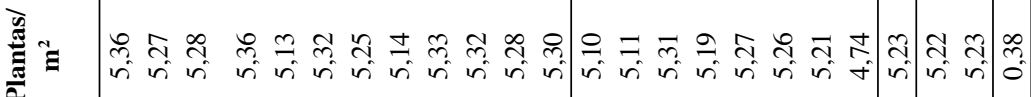

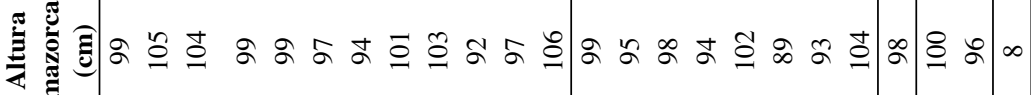

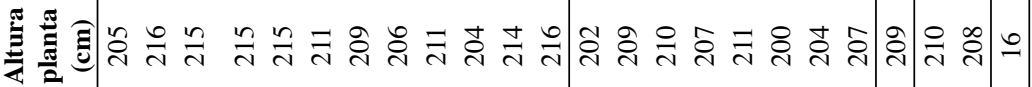

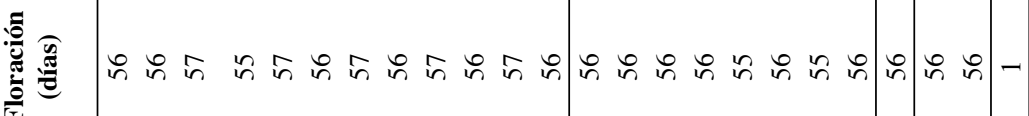

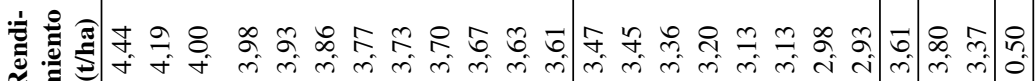

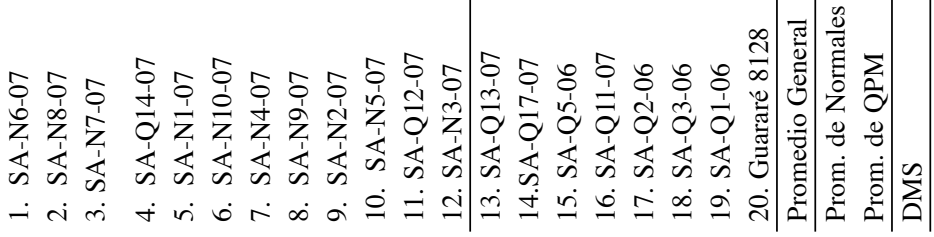




\section{Diferencias entre grupos}

La media de rendimiento de los sintéticos normales superó estadísticamente a los de alta calidad proteica (3,80 vs $3,37 \mathrm{t} / \mathrm{ha}$, respectivamente). A pesar de la ventaja de rendimiento de los maíces normales, los QPM representan una alternativa de producción para los consumidores debido a su mayor contenido de lisina y triptófano con relación a los maíces normales, como lo mencionaron Mertz (1994), Vasal et al. (1994).

Todos los maíces sintéticos normales estuvieron por encima del promedio general, mientras que los QPM sólo el SA-Q14-07 y SA-Q12-07 estuvieron por encima de este valor. El mayor porcentaje de mazorcas podridas fue observado en los sintéticos QPM $(16,1 \%)$, este valor fue diferente estadísticamente al encontrado en los normales $(9,5 \%)$. En relación al tamaño de las mazorcas, el mismo fue superior en los maíces sintéticos normales en comparación a la media de los sintéticos QPM (83,6 vs 73,2 g, respectivamente). En cuanto al número de plantas y mazorcas a la cosecha, así como el número de mazorcas por planta, no se encontraron diferencias significativas entre los dos grupos; lo que sugiere que las diferencias en rendimientos son debido al potencial de rendimiento y adaptabilidad de cada uno de ellos y no debido a la cantidad de plantas cosechadas. En la evaluación del porcentaje de plantas acamadas no se encontraron diferencias altamente significativas entre los dos grupos evaluados, aunque el valor de los maíces normales fue ligeramente superior al encontrado en los sintéticos QPM.

\section{Maíz sintético normal}

Entre los maíces sintéticos normales con mejor rendimiento se destacaron el SA-N6-07, SA-N8-07 y SA-N7-07, con promedios superiores a las 4,0 t/ha. Todos estos sintéticos superaron tanto al testigo de grano normal (Guararé 8128) que tuvo un rendimiento de 2,93 t/ha (Cuadro 5). El testigo Guararé 8128 presentó la población más baja de plantas y mazorcas, debido al alto porcentaje de acame al momento de la floración. Todos los sintéticos presentaron una floración femenina similar, con duración entre 56 y 57 dds. Las medidas de la altura de la mazorca variaron entre 94 a $106 \mathrm{~cm}$, el SA-N4-07 fue el cultivar con la posición más baja de esta variable. El mayor porcentaje de acame se encontró en el testigo normal Guararé 8128 con un $25,5 \%$, mientras que los menores porcentajes lo presentaron el SA-N6-07 y SA-N1-07.

Este año la presión de enfermedades foliares fue baja y las calificaciones en todos los sintéticos estuvieron por debajo de 3,0 (en donde 5,0 es el máximo daño). No se observó diferencias significativas entre las variedades de este ensayo; los sintéticos SA-N6-07 y SA-N7-07 presentaron las calificaciones más bajas entre todos los cultivares evaluados. En relación a la variable que mide el ataque del complejo de hongos de la mazorca (Diplodia maydis y Fusarium sp), los sintéticos normales presentaron valores por debajo del $10 \%$ con excepción del SA-N10-07, SA-N3-07 y Guararé 8128 con promedios de 11,$6 ; 10,8$ y $11,3 \%$, respectivamente.

\section{Sintéticos QPM}

Entre los sintéticos QPM sobresalieron SA-Q1407 y SA-Q12-07 con rendimientos de 3,98 y 3,63 t/ha. El mejor de éstos superó estadísticamente al testigo SA-Q5-06, el cual fue el de mejor rendimiento en el año 2006 (Gordón et al. 2007). Los sintéticos formados en el 2005 (Q14, Q12, Q13, Q17 y Q11) superaron en rendimiento a los maíces sintéticos formados en el 1999 y 2003 (Q5, Q2, Q3 y Q1), lo que indica el avance en la selección de estos nuevos cultivares. No se encontraron diferencias estadísticas entre el número de plantas cosechadas y la altura entre los sintéticos de este tipo de grano. La altura de estos materiales varió entre 202 y $215 \mathrm{~cm}$, el cultivar de menor estatura fue el SA-Q3-06 $(200 \mathrm{~cm})$, seguido por los genotipos SA-Q3-06 y SA-Q1-06. La población de plantas a la cosecha fue superior a las 5,0 plantas $/ \mathrm{m}^{2}$.

En relación al acame de tallo, sobresalió el SA-Q1-06 con un porcentaje de 9,8, el resto de los materiales presentaron acames por encima del $10 \%$. Este mismo cultivar presentó el mayor porcentaje de mazorcas podridas con un $28 \%$, mientras que el menor porcentaje fue presentado por el SA-Q13-07 (10,5\%). En general, esta es una característica que se debe mejorar para evitar pérdidas al productor en ambientes de alta precipitación pluvial. Se debe señalar que estos sintéticos presentaron bajos porcentajes de mazorcas podridas en las localidades de Los Santos, el cual es una provincia con precipitaciones anuales por debajo de los $1200 \mathrm{~mm}$, mientras que en las provincias de Panamá, Darién, Veraguas y la Comarca Ngöbe 
Bugle, las precipitaciones están por encima de los $2500 \mathrm{~mm}$ anuales. En resumen, se identificaron dos sintéticos amarillos normales (SA-N6-07 y SA-N8-07) y dos de alta calidad proteica (SA-Q14-07 y SA-Q1207) que superaron al testigo convencional del área Guararé-8128.

\section{Interacción Genotipo por Ambiente}

El análisis de varianza para la variable rendimiento de grano, así como el valor de los dos primeros ejes del componente principal de la interacción genotipoambiente (PCA), obtenidos a través del modelo AMMI Biplot GGE-SREG se muestra en el Cuadro 6.

Cuadro 6. Análisis de varianza tipo IV y análisis de componentes principales (PCA) para la variable rendimiento de grano de maíz. Instituto de Investigación Agropecuaria de Panamá (IDIAP), Panamá. 2007-2008.

\begin{tabular}{lccccc}
\hline F de V. & g.l. & $\begin{array}{c}\text { Suma de } \\
\text { cuadrados } \\
\text { Tipo IV }\end{array}$ & $\begin{array}{c}\text { Cua- } \\
\text { drados } \\
\text { medios }\end{array}$ & $\begin{array}{c}\text { Prob } \\
>\text { F }\end{array}$ & $\begin{array}{c}\text { \% de la } \\
\text { varianza }\end{array}$ \\
\hline AMB & 5 & 196,12 & 39,22 & 0,001 & 75,9 \\
GEN & 19 & 18,72 & 0,99 & 0,001 & 7,2 \\
GEN x & 95 & 16,16 & 0,17 & 0,001 & 6,3 \\
AMB & & & & & \\
PCA-1 & 23 & 69,01 & 3,00 & 0,001 & 66,0 \\
PCA-2 & 21 & 13,79 & 0,66 & 0,001 & 13,2 \\
Residuo & 51 & 21,83 & 0,43 & 0,001 & 20,8 \\
\hline
\end{tabular}

En La Figura 1 se presenta la gráfica Biplot GGESREG que toma en cuenta las puntuaciones o valores de los dos ejes principales (PCA1 y PCA2), tanto de los 20 genotipos como de los seis ambientes.

Los dos primeros ejes explicaron el 79,2\% de la interacción genotipo ambiente con tan solo el 40,7\% de los grados de libertad. El PCA-1 explicó el 66,0\%, mientras que el PCA-2 fue responsable del 13,2\% con el 21 y $19 \%$ de los grados de libertad, respectivamente.

De acuerdo a Yan et al. (2000), al graficar las puntuaciones de ambos ejes principales, se forma un polígono con los cultivares que quedan en la parte externa de la figura. Este análisis permite agrupar a los ambientes con similar comportamiento, para este

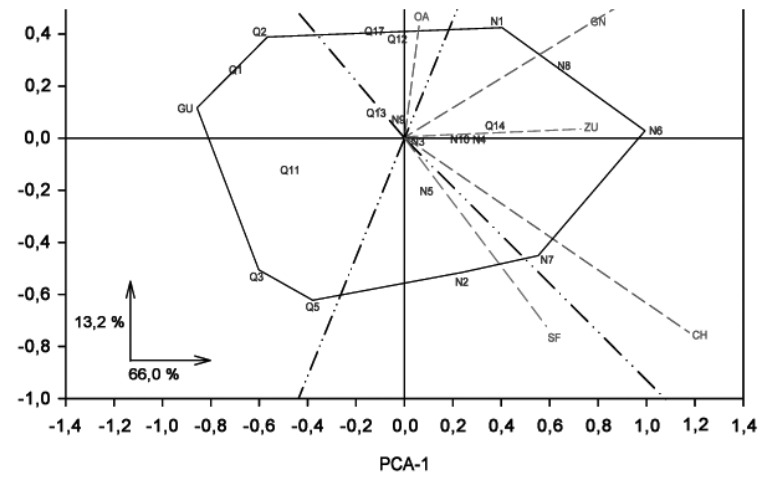

Figura 1. Puntuaciones del primer y segundo eje del componente principal (Biplot-GGE-SReg), de 20 cultivares de maíz evaluados en seis ambientes de Panamá. 2007-2008.

experimento se logró separar tres grupos ambientales El primer grupo (Grupo ambiental A) lo conforman las localidades El Ejido, Chupá, La Zumbona y Llano Jengibre; el promedio de rendimiento de estas localidades fue de 4,39 t/ha. El segundo grupo (Grupo ambiental B) lo constituyó el ensayo sembrado en Ollas Arriba; en esta localidad el rendimiento promedio fue de 1,70 t/ha. El tercer grupo (Grupo ambiental C) estuvo formado por el ensayo sembrado en Santa $\mathrm{Fe}$, en esta localidad la media de rendimiento fue de 2,38 t/ha (Cuadro 7).

De acuerdo a las puntuaciones del eje PCA2, los cultivares más estables fueron el SA-N6-07, SA-Q1407, SA-N10-07 y SA-N4-07 (valores cercanos a cero), mientras que los sintéticos SA-N6-07, SA-N1-07 y SA-N7-07 fueron los que mejor respondieron a los estímulos ambientales por estar en las esquinas del polígono, presentando el mejor rendimiento el sintético SA-N6-07 con 4,44 t/ha) (Yan et al. 2000, Yan et al. 2001). Con relación a la interacción genotipo ambiente los sintéticos que mejor se comportaron en cada uno de los grupos ambientales, se encuentran cerca de cada grupo ambiental (Yan y Hunt 2002, Yan y Rajcan 2002). Ejemplo de esto lo observamos con el sintético SA-N8-07, el cual que tuvo buen comportamiento en las localidades del Grupo A y B (segundo y cuarto lugar con medias de 5,13 y $2,20 \mathrm{t} / \mathrm{ha}$ ) pero ocupó la posición 12 en la localidad del Grupo C con 2,44 t/ha, mientras que el SA-Q5-06 fue el de tercer mejor rendimiento $(2,95 \mathrm{t} / \mathrm{ha})$ mejor en los ambientes del Grupo C, pero su rendimiento quedo en las posiciones 16 y 15 en los ambientes del Grupo A y B $(3,94$ 
Cuadro 7. Rendimiento de grano de los sintéticos de maíz evaluados según grupo ambiental. Panamá. 2007-2008.

\begin{tabular}{|c|c|c|c|c|c|c|c|}
\hline \multirow[b]{2}{*}{ Sintéticos } & \multicolumn{4}{|c|}{ Localidades del Grupo A } & \multirow{2}{*}{$\begin{array}{c}\text { Grupo A } \\
\text { Promedio }\end{array}$} & \multirow{2}{*}{$\begin{array}{c}\text { Grupo B } \\
\text { Ollas Arriba }\end{array}$} & \multirow{2}{*}{$\begin{array}{l}\text { Grupo C } \\
\text { Santa Fe }\end{array}$} \\
\hline & El Ejido & Chupá & La Zumbona & Llano Jengibre & & & \\
\hline 1. SA-N1-07 & 6,33 & 4,48 & 4,36 & 4,18 & $4,84^{4}$ & 1,76 & 2,48 \\
\hline 2. SA-N2-07 & 5,40 & 5,44 & 4,03 & 3,60 & 4,62 & 1,07 & 2,64 \\
\hline 3. SA-N3-07 & 5,83 & 4,48 & 3,94 & 3,38 & 4,41 & 1,42 & 2,58 \\
\hline 4. SA-N4-07 & 6,03 & 4,99 & 4,01 & 3,84 & 4,72 & 1,30 & 2,46 \\
\hline 5. SA-N5-07 & 5,56 & 4,83 & 3,89 & 3,49 & 4,44 & 1,57 & 2,66 \\
\hline 6. SA-N6-07 & 6,92 & 5,94 & 4,62 & 4,27 & $5,44^{1}$ & 1,95 & $2,97^{2}$ \\
\hline 7. SA-N7-07 & 6,02 & 5,44 & 4,22 & 4,06 & $4,93^{3}$ & 1,03 & $3,26^{1}$ \\
\hline 8. SA-N8-07 & $6,42^{2}$ & $5,34^{4}$ & $4,62^{2}$ & $4,13^{3}$ & $5,13^{2}$ & $2,20^{4}$ & $2,44^{12}$ \\
\hline 9. SA-N9-07 & 5,34 & 4,41 & 3,65 & 3,85 & 4,31 & $2,36^{1}$ & $2,77^{4}$ \\
\hline 10. SA-N10-07 & 5,65 & 4,77 & 4,10 & 3,95 & 4,62 & 1,93 & 2,75 \\
\hline 11. SA-Q11-07 & 4,45 & 4,33 & 3,57 & 3,64 & 4,00 & 1,39 & 1,81 \\
\hline 12. SA-Q12-07 & 5,47 & 4,72 & 4,07 & 3,67 & 4,48 & $2,25^{2}$ & 1,58 \\
\hline 13. SA-Q13-07 & 5,57 & 4,61 & 3,48 & 3,41 & 4,27 & 1,68 & 2,05 \\
\hline 14. SA-Q14-07 & 5,87 & 5,20 & 4,13 & 3,90 & 4,78 & $2,22^{3}$ & 2,54 \\
\hline 15. SA-Q1-06 & 5,00 & 3,64 & 3,16 & 3,01 & 3,70 & 1,48 & 1,61 \\
\hline 16. SA-Q3-06 & 4,47 & 4,73 & 3,03 & 2,33 & 3,64 & 2,06 & 2,18 \\
\hline 17. SA-Q17-07 & 5,71 & 4,09 & 4,02 & 3,59 & 4,35 & 1,48 & 1,83 \\
\hline 18. SA-Q2-06 & 5,34 & 3,30 & 3,19 & 3,12 & 3,74 & 1,75 & 2,10 \\
\hline 19. Guararé 8128 & 4,15 & 3,23 & 3,52 & 3,21 & 3,53 & 1,64 & 1,83 \\
\hline 20. SA-Q5-06 & $4,37^{19}$ & $4,27^{16}$ & $4,15^{5}$ & $2,97^{19}$ & $3,94^{16}$ & $1,48^{15}$ & $2,95^{3}$ \\
\hline Promedio & 5,50 & 4,61 & 3,89 & 3,58 & 4,39 & 1,70 & 2,38 \\
\hline
\end{tabular}

* El superíndice indica la posición de acuerdo al orden de los rendimientos.

y 1,48 t/ha, respectivamente). Este comportamiento se puede observar al comparar las medias presentadas en el Cuadro 7, el cual resume el rendimiento general y por cada grupo ambiental. El modelo Biplot GGESReg, resultó apropiado para estimar con precisión los patrones de respuesta de los sintéticos así como de los efectos ambientales.

\section{AGRADECIMIENTO}

Los autores desean manifiestan su agradecimiento a la Secretaría Nacional de Ciencia Tecnología e Innovación (SENACYT) por su apoyo financiero. A los ingenieros Walker González, Omar Alfaro y Felipe González por su desinteresada participación en la conducción de algunos de los ensayos de esta investigación.

\section{LITERATURA CITADA}

Becker, HC. 1981. Correlation among some statistical measure of phenotypic stability. Euphytica 30: 835-840.

Becker, HC; Leon, J. 1988. Stability analysis in plant breeding. Plant Breeding 101:1-23.

CIMMYT (Centro Internacional de Mejoramiento de Maíz y Trigo). 1999. Informe Anual 1999-2000. (en línea). Consultado 15 set. 2009. Disponible en www.cimmyt. org/whatiscimmyt/AR99_2000Spa/contenido.htm

CIMMYT (Centro Internacional de Mejoramiento de Maíz y Trigo). 2009. México y el CIMMYT (en línea). Consultado 30 oct. 2009. Disponible en http://www. cimmyt.org/spanish/wps/mexico/mexicocimmyt.htm

Crossa, J. 1988. A comparison of results obtained with two methods for assessing yield stability. Theor. Appl. Genet 75:460- 467.

AGRONOMÍA MESOAMERICANA 21(1):11-20. 2010 
Crossa, J. 1990. Statistical analysis of multi location trials. Advances in agronomy 44:55-85.

Crossa, J; Gauch, Jr, HG; Zobel, RW. 1990. Additive main effects and multiplicative interaction analysis of two international maize cultivar trials. Crop. Sci. 30:493500 .

Crossa, J; Fox, PN; Pfeiffer, WH; Rajaram, S; Gauch, Jr. HG 1991. AMMI adjustment for statistical analysis of an international wheat yield trial. Theor Appl. Genet. 81:27-37.

Cubero, JI. 2003. Introducción a la mejora genética vegetal. Ediciones Mundi Prensa. 2 ed. Madrid España. 567 p.

Díaz-Romeu, R; Hunter, A. 1978. Metodología de muestreo de suelos y tejidos vegetal e investigación en invernadero. Turrialba, Costa Rica, CATIE. 68 p.

Eberhart, SA; Russell, WA. 1966. Stability parameters for comparing varieties. Crop. Sci. 6:36-40.

FAO (Food and agriculture organization). 1993. El maíz en la nutrición humana. Colección FAO: Alimentación y nutrición No. 25 (en línea). Consultado 15 set. 2009. Disponible en www.fao.org/docrep/t0395s/T0395S03.htm

Finlay, KW; Wilkinson, GN. 1963. The Analysis of Adaptation in plant breeding program. Aust. J. Agric. Res. 14:742-754.

Gauch, HG; Zobel, RW. 1988. Predictive and postdictive success of statistical analyses of yield trials. Theor. Appl. Genet. 76:1-10.

Gordón, R; Camargo, I; Franco, J; Quirós, E. 2007. Evaluación de nueve sintéticos de maíz de alta calidad proteica en 14 localidades, Panamá. 2006-2007. Informes técnicos del POA 2006-2007. IDIAP. 96 p.

Krivanek, AF; De Groote, H; Gunaratna, NS; Diallo, AO; Friesen, D. 2007. Breeding and disseminating quality protein maize (QPM) for Africa. African Journal of Biotechnology 6(4):312-324.

Lin, CS; Binns, MR; Lefkovith, LP. 1986. Stability Analysis. Where do we stand? Crop Sci. 26:894-900.

Mendoza, E; Andrio, E; Juarez, JM; Mosqueda, C; Latournerie, L; Castañón, G; López, A; Moreno, E. 2006.
Contenido de Lisina y Triptófano en genotipos de maíz de alta calidad proteica y normal. Universidad y Ciencia, Trópico Húmedo 22(2):153-161.

Mertz, EE. 1994. Thirty years of opaque 2 maize. In: Quality protein maize. 1964-1994. Proc. of Symp. of Quality Protein Maize. EMBRAPA/CNPMS, Sete Lagoas M. G. Brasil. p. 1-10.

MEF (Ministerio de Economía y Finanzas). 2005. La pobreza en Panamá. Encuesta de niveles de vida. 2003. Principales resultados. $9 \mathrm{p}$.

Ortega CA; Cota, AO; Vasal, SK; Villegas, ME; Córdoba, $\mathrm{OH}$; Barreras, SMA; Wong, PJJ; Reyes, MCA; Preciado, ORE; Terrón, IA; Espinoza, CA. 2001 H-441C, H-442C y H-469C, híbridos de maíz de calidad proteínica mejorada para el Noroeste y subtrópico de México. Ed. INIFAP. Folleto Técnico No. 41:4-15.

Perkins, JM; Jinks, JL. 1968. Environmental and genotypeenvironmental components of variability. IV Nonlinear interactions for multiple inbred lines. Heredity, 23:525-535.

Vasal, SK; Vergara, AN; Mc Lean, S. 1994. Estrategias en el desarrollo de híbridos tropicales de maíz. Agronomía Mesoamericana 5:184-189.

Westcott, B. 1986. Some methods of analyzing genotype environment interaction. Heredity 56:243-253.

Yan, W, L; Hunt, A; Sheng, Q; Szlavnics, Z. 2000. Cultivar evaluation and mega environment investigation based on the GGE Biplot. Crop Sci. 40:597-605.

Yan, W; Cornelius, P; Crossa, J; Hunt, LA. 2001. Two types of GGE Biplots for analyzing multi - environment trial data. Crop Sci. 41:656-663.

Yan, W; Hunt, A. 2002. Biplots analysis of multi - environment trial data. $\mathrm{CAB}$ International. Quantitative Genetics, Genomics and Plant Breeding. Ed. M.S.Kang. p. 289-303.

Yan, W; Rajcan, I. 2002. Biplot analysis of test sites and trait relations of soybean in Ontario. Crop Sci. 42:11-20.

Zobel, RW; Wright, MJ; Gauch, HG. Jr. 1988. Statistical analysis of a yield trial. Agron. J. 80:388-393. 